\title{
Square-wave switching by crossed-polarization gain modulation in vertical-cavity semiconductor lasers
}

\author{
J. Mulet, M. Giudici, ${ }^{*}$ J. Javaloyes, and S. Balle \\ Institut Mediterrani d'Estudis Avançats, CSIC UIB, E-07071 Palma de Mallorca, Spain
}

(Received 9 March 2007; published 1 October 2007)

\begin{abstract}
We study experimentally and theoretically the effects of crossed-polarization reinjection (XPR) on the output characteristics of a vertical-cavity semiconductor laser. We find a set of parameters values for which each polarization component develops a square-wave modulation at a period close to twice the reinjection delay. We analyze the regularity of this modulation in terms of the laser pumping current and of the reinjection level. These observations are numerically reproduced within the spin-flip model modified to account for XPR. In particular, the degradation of the square-wave switching is linked to the finite value of the spin-flip rate, and it occurs when the current approaches the boundaries of polarization bistability.
\end{abstract}

DOI: 10.1103/PhysRevA.76.043801

PACS number(s): 42.55.Px

\section{INTRODUCTION}

Semiconductor lasers are prone to develop a variety of dynamical behaviors, such as oscillations in the total power, mode partition or mode hopping. The origin of such dynamical behaviors can often be traced back to the nonlinear optical response of the active material, which arises from the changes induced by the optical field in both the total carrier density and its distribution over the energy bands, hereby changing the gain and refractive index of the semiconductor on fast time scales. This tendency can be reduced or further enhanced by appropriate design of the laser architecture, which allows to tailor the laser modes as well as their mutual coupling and their degree of interaction with the active medium.

In order to passively produce optical pulses at high repetition frequencies several strategies have been devised. Most of them are based on saturable absorbers $[1,2]$ that lead to either passive $Q$-switching or to mode-locking. Another proposal involves polarization-rotated optical feedback [3], where the two linearly polarized components, LP- $x$ and LP- $y$, of the light emitted by the device are fed back into the laser cavity after the LP- $x$ component is converted into the LP- $y$ component and vice versa. This scheme for obtaining high-speed optical pulses has been applied to edge-emitting lasers by Kawaguchi et al. [3] and to vertical-cavity semiconductor lasers (VCSELs) by Jiang et al. [4] and Li et al. [5].

More recently, crossed-polarization reinjection (XPR) [6-8] has been proposed for generating optical pulses. In such scheme, the emission from the laser is split into its two linearly polarized components, LP- $x$ and LP- $y$. Then, only one of these components (LP- $x$, say) is converted into the orthogonal polarization component (LP- $y$, say) and then it is injected back into the laser cavity. In this way, the photon density in each of the polarization components is modified through cross-saturation, eventually leading to pulsed emis-

\footnotetext{
*Permanent address: Institut Non Linéaire de Nice, Université de Nice Sophia Antipolis-Centre National de la Recherche Scientifique, 1361 route des lucioles, F-06560 Valbonne, France
}

sion [7]. In some operating ranges, square-wave modulation of the power emitted in each of the polarization orientations with a period close to twice the delay introduced by the XPR arm can be obtained $[6,8]$. These features have recently been experimentally demonstrated in edge-emitting lasers [8], thus stimulating the search for other potential configurations capable of further exploiting the cross-saturation effects among orthogonal polarization components in lasers.

Such effects are expected to be highly relevant in VCSELs. These devices are intrinsically single-longitudinal mode, and they have nearly degenerate polarization orientations owing to their almost perfect symmetry around the cavity axis. The polarization modes have slightly different losses and frequencies due to the residual dichroism and birefringence in the cavity, and they exhibit a high degree of coupling because both have the same spatial profile and share the same active medium. These characteristics allow for the existence of complicated polarization properties in the emission from VCSELs, which may display polarization switching accompanied in some cases by regions of polarization bistability [9] or even regimes where the polarization of the output exhibits complicated dynamics [10].

In addition, the quasidegeneracy of orthogonal polarization states in VCSELs enables efficient cross-gain modulation among orthogonal polarizations when the device is used as an optical amplifier [11]. In principle, these characteristics should also allow for efficient XPR. Recently, the authors have proposed to combine XPR with conventional optical feedback [12] in order to achieve passive mode-locking in VCSELs. However, one might wonder to which extent the dynamics of VCSELs with XPR would be the same as in edge-emitting lasers, because the small dichroism and birefringence of the latter allow for polarization bistability as well as polarization instabilities that are not observed in the former.

In this paper, we investigate experimentally and theoretically the effects of XPR on the output characteristics of VCSELs. We show that, for a VCSEL that does not display any polarization instabilities when solitary, the output power develops a square-wave modulation when subject to XPR. The period of the square waveform is close to twice the reinjection delay. The onset of this square-wave regime de- 


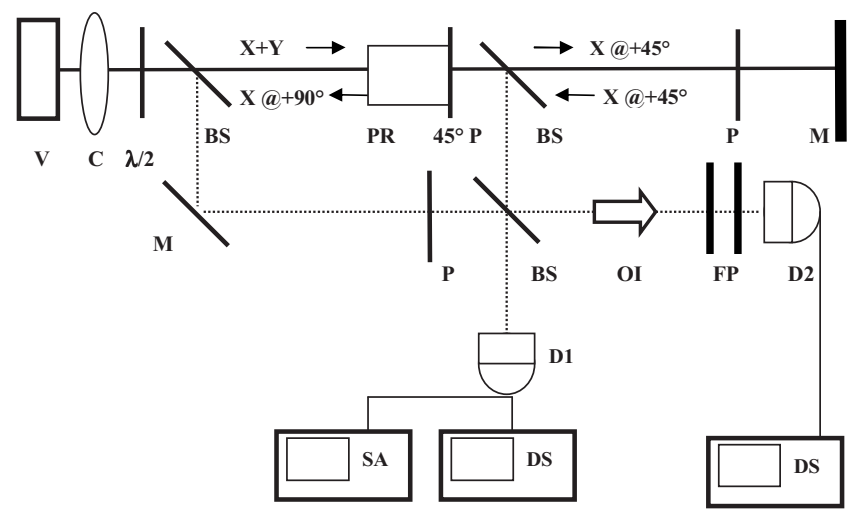

FIG. 1. Experimental setup. $V$ : Vertical cavity surface emitting laser. $C$ : Collimator. $\lambda / 2: \lambda / 2$ waveplates. PR: $+45^{\circ}$ polarization rotator. $45^{\circ} \mathrm{P}$ polarizer placed at $+45^{\circ}$ with respect to VCSEL $x$ polarization axis. $P$ : Polarizer. $D 1$ : fast detector. $D 2$ : Detector. OI: Optical isolator. FP: Fabry-Pérot resonator. DS: Digital scope. SA: Spectrum analyzer. BS: Beam splitter.

pends on both the current and level of XPR. For a fixed current, square-wave switching is observed for intermediate levels of XPR; lower levels of XPR lead to an irregular output power, while a deterioration of the square waves is observed for higher levels of XPR. A similar scenario is found while varying the current for a fixed (intermediate) level of XPR. For currents close to the laser threshold the output is quite irregular, but square waves develop when the current is increased; however, a deterioration of the square-wave switching is observed when current is further increased. The onset of the square-wave regime as the level of XPR is increased can be reproduced with a model of the type used in [8] even if this type of model was originally conceived for edge-emitting lasers. However, in its original form, the model in [8] does not reproduce the subsequent degradation of the square-wave regime. Instead, both the onset and subsequent degradation of the square-wave regime can be reproduced within the spin-flip model (SFM) [13], suitably modified to account for XPR along the lines given in [8]. The SFM is a model specifically developed for studying polarization dynamics in VCSELs. As compared to the model used in [8], the SFM includes not only the dynamics of the total carrier density, but also that of the population difference between the two spin channels. Depending on parameter values, the SFM displays polarization instabilities or polarization bistability $[10,15]$ that can never occur in the model of [8]. In the limit of infinite spin relaxation rate, the SFM reduces to the model in [8]; otherwise, there is cross saturation between the polarizations [17] that, as we shall see, allows us to explain the degradation of the square-wave regime observed in our experiment.

\section{EXPERIMENTAL RESULTS}

\section{A. Experimental setup}

Our experimental setup is schematically shown in Fig. 1. It comprises a VCSEL (V) that is thermally stabilized to better than $0.01{ }^{\circ} \mathrm{C}$ and driven by a very stable current power supply (ILX LDC3714B, stable to better than $0.01 \mathrm{~mA}$ ). The output of the VCSEL is collimated by a collimator $(\mathrm{C})$ and sent through a $\lambda / 2$ waveplate followed by a nonpolarizing beam splitter (BS). The waveplate allows for careful alignment of the polarization directions with the axis of the optical elements of the setup. The beam is sent through a $45^{\circ}$ polarization rotator $(\mathrm{PR})$ with a linear polarizer $(P)$ at the output placed such that only the LP- $x$ component of the input beam can pass through. This beam is reflected back by a mirror $(M)$ placed at a distance $L$ and sent back to the VCSEL upon passing again through the polarization rotator. In this way, the LP- $x$ component of the light is fed back into the VCSEL cavity after being aligned to the $y$ polarization axis. Instead, the LP- $y$ component is suppressed by the linear polarizer placed after the polarization rotator (extinction ratio of $40 \mathrm{~dB}$ ). Another polarizer is placed in front of the external mirror in order to control the level of XPR. In this setup, the maximum level of XPR achieved corresponds to $10 \%$ of the light in the LP- $x$ polarization. This fraction has been measured opening the reinjection loop before the VCSEL collimator and therefore it does not include the coupling losses with the VCSEL.

Two additional beam splitters are inserted in the setup in order to extract two signals for monitoring each polarization component. One beam passes through a polarizer in order to obtain the LP-y component, while the second beam comes from the feedback circuit and therefore it contains only the LP- $x$ component. The two beams are sent to a $8 \mathrm{GHz}$ bandwidth detector ( $D 1$, Thorlabs PDS8) where each polarization can be monitored separately by cutting the other beam. The polarization signals are monitored with a $2 \mathrm{GHz}$ oscilloscope (Lecroy WavePro 960) and a $8.5 \mathrm{GHz}$ spectrum analyzer (Anritsu MS2602A). We detect also the polarization resolved optical spectra by using a scanning Fabry Pérot (D2).

\section{B. Results}

The experimental results reported here were obtained with a proton-implanted VCSEL manufactured by the Vixel Corporation, lasing at $850 \mathrm{~nm}$ with an active area of diameter around $8 \mu \mathrm{m}$. We have tried several samples and we have noticed that one of the crucial parameters in order to obtain appreciable effects when applying XPR is the birefringence of the device. We have observed that for VCSELs having birefringence larger than $10 \mathrm{GHz}$ the effects of the reinjection are negligible even when the reinjection strength is set at the largest value.

The polarization-resolved light intensity output versus pumping current curve of solitary VCSEL reveals that the laser threshold occurs at $2.8 \pm 0.2 \mathrm{~mA}$. The emission is linearly polarized up to $5 \mathrm{~mA}$, where multitransverse mode operation starts. The birefringence is $5.0 \pm 0.3 \mathrm{GHz}$, and the VCSEL emits in the high-frequency mode, which we denote as LP- $x$ component.

Figure 2 shows the time behavior of the VCSEL with XPR when the $\lambda / 2$ waveplate is set to allow the LP- $y$ component to pass through the polarization rotator and the polarizer. The pumping current $(J)$ is fixed at $J=3.5 \mathrm{~mA}$, the reinjection delay $(\tau)$ is of $(2 L) / c=\tau \approx 4 \mathrm{~ns}$, while the 

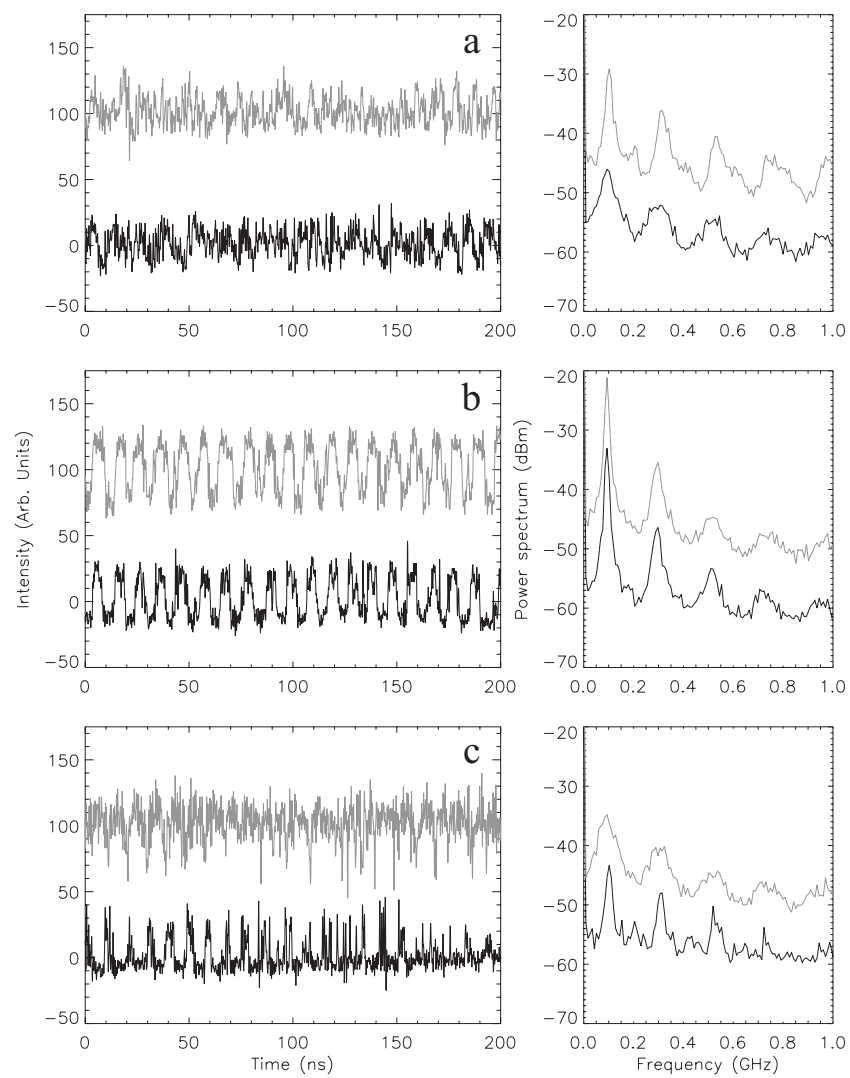

FIG. 2. $x$-polarization (black curve) and y-polarization (gray curve) output of the reinjected VCSEL: Time series (left panels) and power spectrum (right panels). In the time series the gray curve has been displaced vertically by 100 units, while in the power spectra it has been displaced vertically by $10 \mathrm{dBm}$. Three different levels of reinjection: (a) $0.2 \%$, (b) $1 \%$, and (c) $10 \%$. The signals are amplified and $\mathrm{AC}$ coupled to the scope.

reinjection level is varied through the polarizer in front of the mirror (see Fig. 1).

For low reinjection rate, the power spectra of each polarization display broad peaks. The first peak is located at $100 \mathrm{MHz}$, i.e., slightly below the inverse of $2 \tau$, while the other peaks appear at the odd multiples of this frequency. The time signals are rather irregular. The LP- $x$ component fluctuates around an average value which is lower than that of the solitary laser emission. Instead, the LP- $y$ component is activated by XPR and therefore fluctuates at a level which is higher than that in the solitary laser.

Upon increasing XPR level, a square waveform becomes better and better defined in the time signal. Accordingly, the peaks in the power spectrum increase and sharpen. The most regular time series has been obtained for XPR levels $\sim 1 \%$. Even in this case, we see the even peaks in the power spectra which indicate that the square waveform is not perfect. In this regime, the power in the LP- $x$ component switches from the level corresponding to solitary operation to spontaneous emission level. The LP-y component displays a similar switching but in antiphase with LP- $x$ emission. This antiphase dynamics cannot be seen in Fig. 2 because of the two polarization signals have not been monitored simultaneously.
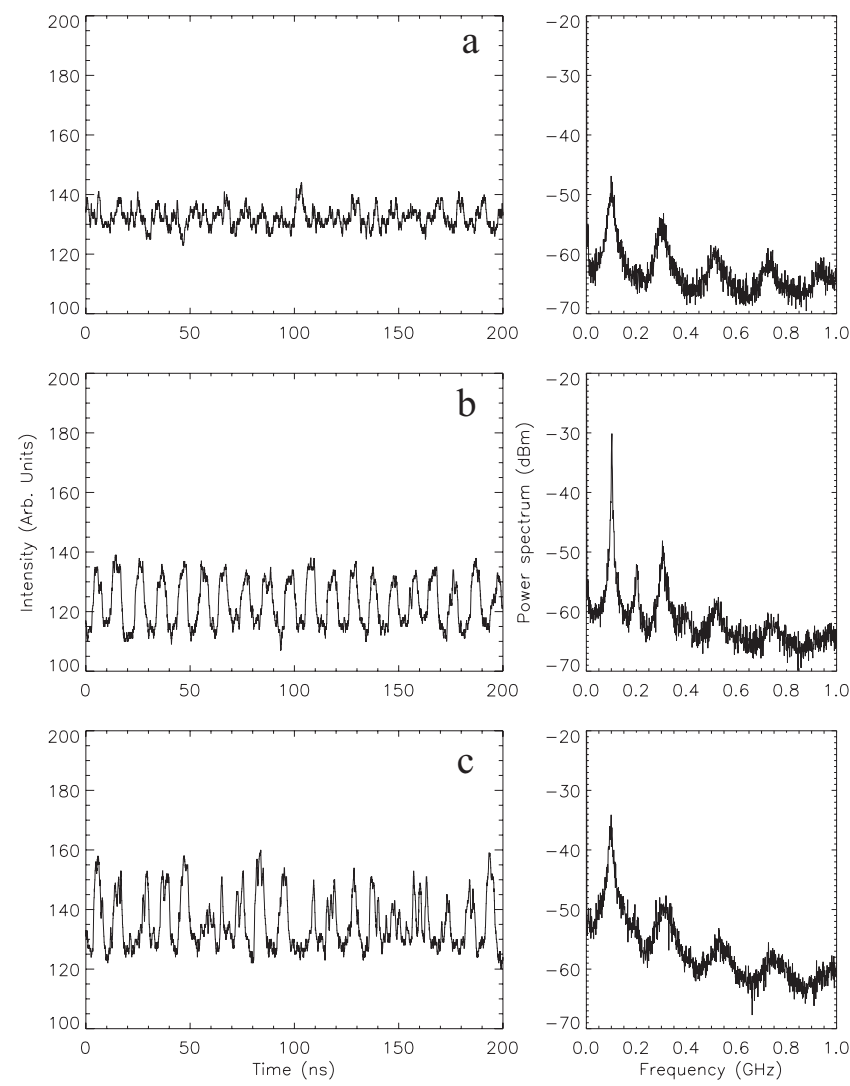

FIG. 3. $x$-polarization output of the reinjected VCSEL: Time series (left panels) and power spectrum (right panels). Three different levels of pumping current for reinjection fixed at 1\%: (a) $J$ $=1.125 J_{\text {th }}$, (b) $J=1.25 J_{\text {th }}$, and (c) $J=1.55 J_{\text {th }}$.

However, antiphase dynamics is confirmed since the total intensity remains constant in this regime.

If the level of XPR is further increased, the regularity of the square waveform worsens. The LP- $x$ component displays irregularly distributed upward pulses instead of square waveforms. Its average intensity value further decreases. Instead, the LP- $y$ component increases its average intensity and it exhibits downward irregular pulses in antiphase with those in the LP- $x$ component.

A similar scenario is found while keeping a constant level of XPR and varying the current. Figure 3 shows the polarization resolved traces and power spectra for three different values of the current for the XPR level that provided the optimal square waveform in Fig. 2. These results demonstrate that there exists also an optimal current for the regularity of the square waveform. For both low and too high values of $J$, the square waveform is degraded in comparison with this optimal current.

For the optimal current, Fig. 4 shows the polarizationresolved power and optical spectra of both the solitary laser and with the optimal XPR level. For the solitary laser, the power spectrum does not display any structure apart from the relaxation oscillations that appear strongly damped around $3 \mathrm{GHz}$. When the XPR is applied, the low frequency part of the power spectra (below $1 \mathrm{GHz}$ ) of both polarizations almost coincide and they display multiple peaks as already 

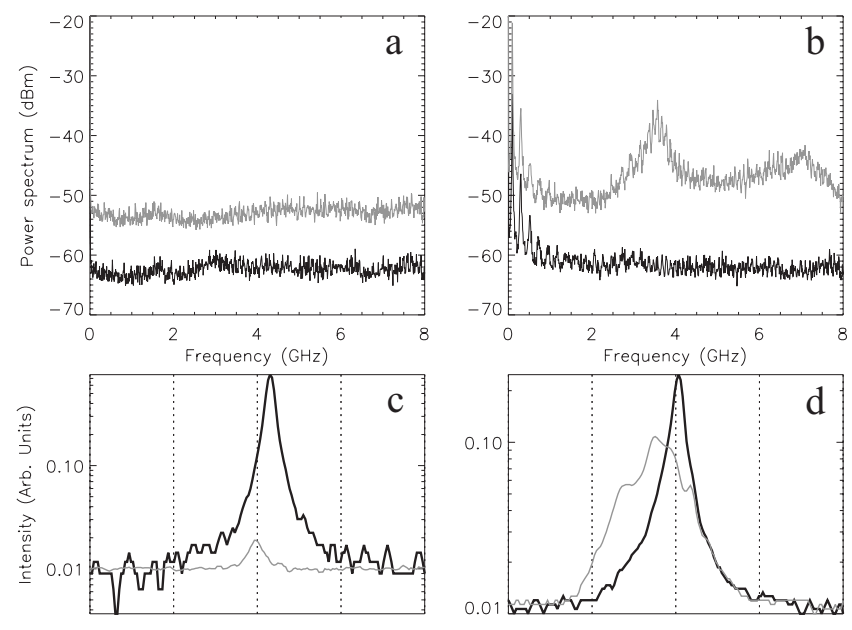

Frequency $(15 \mathrm{GHz} /$ div)

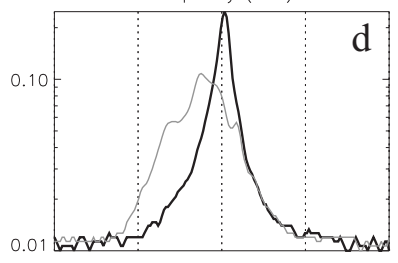

Frequency $(15 \mathrm{GHz} /$ div)

FIG. 4. Comparison between the solitary laser emission and the XPR injected laser. (a) Power spectra of the $x$-polarization component and of the $y$-polarization component (black and gray lines, respectively). The gray line is also vertically shifted of $10 \mathrm{dBm}$ for clarity. $J=1.25 J_{\text {th }}$. (b) As in (a) but for XPR reinjected VCSEL; parameters are the same of Fig. 2 central panel $J=1.25 J_{\text {th }}$ and XPR reinjection level fixed at $1 \%$. (c) Optical spectra of the $x$ and $y$ polarization components (black and gray lines, respectively). $J$ $=1.25 J_{\text {th }}$. (d) Same as in (c) but for XPR reinjected VCSEL; parameters are the same of Fig. 2 central panel $J=1.25 J_{\text {th }}$ and XPR reinjection level fixed at $1 \%$.

discussed. At higher frequencies, the power spectrum of the LP- $x$ component displays low noise and only a weak peak at $\sim 3 \mathrm{GHz}$ can be identified. This frequency component is close to the relaxation oscillation frequency (ROF) of the solitary VCSEL. On the contrary, the power spectrum of the LP-y component displays larger noise levels with a strong peak at $3.5 \mathrm{GHz}$ and its harmonic at $7 \mathrm{GHz}$. The first peak is located between the ROF and birefringence frequencies. This effect can be understood as intracavity amplification of the reinjected field, which is detuned from the LP-y cavity resonance thus yielding frequency pulling in the spectrum. The optical spectra of the solitary laser show dominant emission in LP- $x$ component, in addition to a weak incoherent emission in LP- $y$. When the XPR is applied, the optical spectra show that the LP- $x$ emission is strongly suppressed. This suppression can be as large as $13 \mathrm{~dB}$ for the strongest XPR level $(10 \%$ of the light in the LP- $x$ component is returned in the LP- $y$ component). In contrast, as the level of XPR is increased, the peak corresponding to LP-y emission increases its power. At the same time, it broadens and displays shoulders that correspond to the peaks observed in the power spectrum.

Finally, it is worth mentioning that all the above results are insensitive to wavelength-scale variations of the optical path, and essentially the same sequence of dynamic behaviors is obtained for reinjection times $\tau$ of the order of ns. However, we could not study either the limit of short reinjection delays because of the size of the optical isolator, or that of long reinjection delays because of the reduction in coupling efficiency.

\section{MODEL AND NUMERICAL RESULTS}

\section{A. Model}

In order to theoretically analyze the above experimental findings, we use the so-called spin-flip model (SFM), suitably modified for incorporating the effects of XPR. The direct way to incorporate XPR is in terms of the linearly polarized components of the field, $E_{x, y}$. In this linearly polarized base, assuming that the LP- $x$ is sent back into the LP- $y$, the equations for the field amplitudes read [15]

$$
\begin{gathered}
\dot{E}_{x}=(1+i \alpha)\left[(N-1) E_{x}+i n E_{y}\right]-z E_{x}, \\
\dot{E}_{y}=(1+i \alpha)\left[(N-1) E_{y}-i n E_{x}\right]+z E_{y}+\beta E_{x}^{\tau},
\end{gathered}
$$

where $N(n)$ is the scaled total (difference of) carrier density in the two spin channels. In addition, $\alpha$ stands for the linewidth enhancement factor [14] and $z=\left(\gamma_{a}+i \gamma_{p}\right)$ describes via $\gamma_{a}\left(\gamma_{p}\right)$ the linear dichroism (birefringence) of the VCSEL cavity. The term $E_{x}^{\tau}=E_{x}(t-\tau)$ in the equation for LP- $y$ describes the effects of XPR after a time delay $\tau$. Notice that in this scheme, the validity of the model is not restricted to small values of $|\beta|$. It is also worth noticing that the reinjection phase is irrelevant to the dynamics only in the limit of infinitely fast spin-flip decay rate, i.e., whenever $n=0$, thus the reinjection phase can be gathered in a global phase by a redefinition of the fields.

The natural representation for the SFM is in terms of the left- and right-circularly polarized components of the optical field $E_{ \pm}=\left(E_{x} \pm i E_{y}\right) / \sqrt{2}$. In terms of $E_{ \pm}$, the model reads $[13,15]$

$$
\begin{gathered}
\dot{E}_{ \pm}=(1+i \alpha)(N \pm n-1) E_{ \pm}-z E_{\mp} \pm i \frac{\beta}{2}\left(E_{+}^{\tau}+E_{-}^{\tau}\right), \\
T \dot{N}=\mu-N-(N+n)\left|E_{+}\right|^{2}-(N-n)\left|E_{-}\right|^{2}, \\
T \dot{n}=-\gamma_{s} n-(N+n)\left|E_{+}\right|^{2}+(N-n)\left|E_{-}\right|^{2} .
\end{gathered}
$$

In these equations, time has been scaled to the cavity decay rate $\kappa$ while $T=\kappa / \gamma_{e}$ represents the scaled carrier lifetime and $\gamma_{s}$ is the ratio of the spin-flip and carrier relaxation rates. $\mu$ represents the rate of carrier density injected into the active region due to the bias current. Finally, we have added to the time evolution independent Langevin sources $\left(F_{+}(t), F_{-}(t), F_{N}(t), F_{n}(t)\right)$, describing spontaneous emission noise, whose properties are described in Ref. [16]. The spontaneous emission factor amplitude is $\eta_{\mathrm{sp}} \sim 10^{-4}-10^{-2}$.

The SFM reduces to the model in [8] when the spin relaxation rate $\gamma_{s} / T$ is infinite. Large but finite values of $\gamma_{s} / T$ provide cross saturation between the polarizations [17] that may lead, depending on the parameter values, to polarization instabilities or polarization bistability $[10,15]$. In our case, the spin-relaxation process has important dynamical effects on the square-wave switching, and in particular it leads to the deterioration of the square-wave regime. 

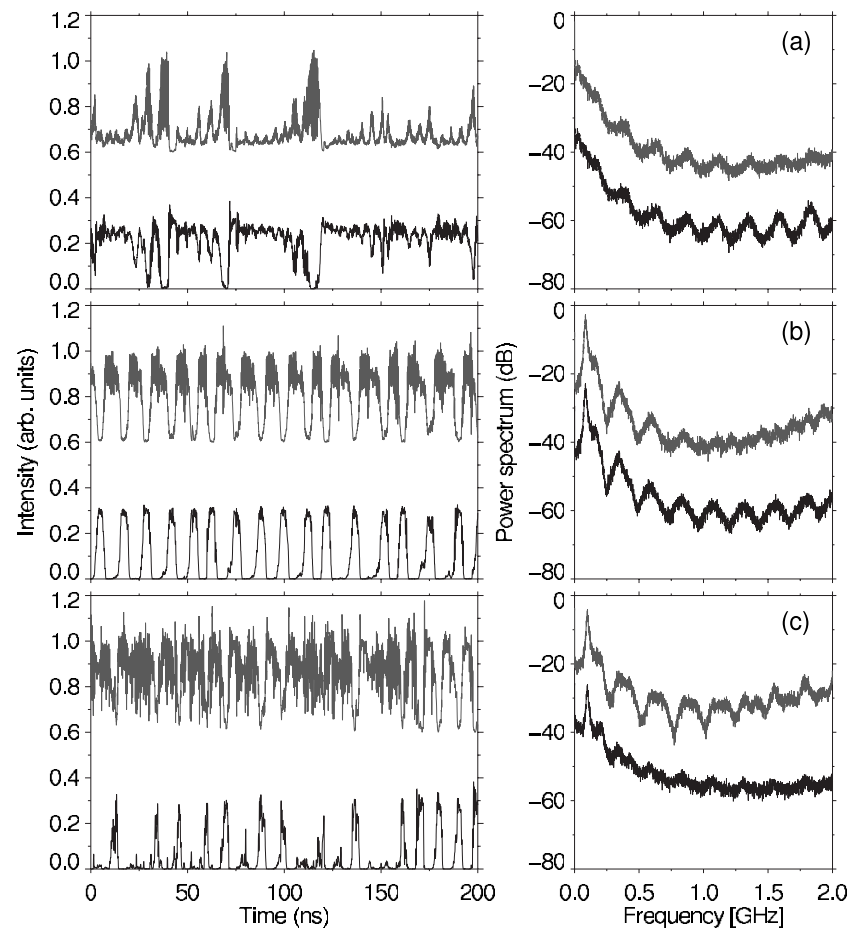

FIG. 5. (Left panels) Intensity of the LP-x (black) and LP-y (gray) components of the VCSEL under different levels of XPR: (a) $\beta=0.04$, (b) $\beta=0.1$, and (c) $\beta=0.25$. The traces of the LP- $y$ component have been vertically shifted for the sake of clarity. (Right panels) The corresponding power spectra of the time traces. $\eta_{\mathrm{sp}}$ $=10^{-3}$ and $\mu=1.25$.

\section{B. Numerical results}

We adjust the parameters of the model according to the experimental conditions. We consider standard values for the linewidth enhancement factor $\alpha=2$, the cavity decay rate $\kappa$ $=300 \mathrm{~ns}^{-1}$, the carrier lifetime $\gamma_{e}=2 \mathrm{~ns}^{-1}(T=150)$, and the spin-flip rate $150 \mathrm{~ns}^{-1}\left(\gamma_{s}=75\right)$. Since the dominant mode is on the blue side of the optical spectrum, we have $\gamma_{a}<0$ and $\gamma_{p}<0$. The birefringence $\gamma_{p}$ is matched to the experimentally observed frequency splitting between the $x$ and $y$-polarized emission, which is about $4.8 \mathrm{GHz}$, thus $\gamma_{p}$ $=-5.24 \times 10^{-2}$. The linear dichroism is taken as $\gamma_{a}=-2.6$ $\times 10^{-3}$ and the spontaneous emission factor is $\eta_{\mathrm{sp}} \sim 10^{-3}$.

For these parameter values, even in the presence of high noise levels $\eta_{\mathrm{sp}} \sim 10^{-2}$, the solitary VCSEL does not present any polarization instability upon increasing the injection current from threshold $\mu_{\mathrm{th}}=\left(1+\gamma_{a}\right)$ up to $\mu_{\max }=2 \mu_{\mathrm{th}}$, and it always lases on the LP- $x$ component, which is the one with the highest frequency. We have found that our results are quite insensitive to the reinjection phase. Therefore, we take real positive values of $\beta$.

Figure 5 shows the behavior of the VCSEL for different levels of XPR while keeping a constant bias current at $\mu$ $=1.25$, which yields a ROF $\nu_{\mathrm{ROF}} \sim 3 \mathrm{GHz}$ as in the experiment. For very low injection levels, the VCSEL output is stable and elliptically polarized. The main axis of the ellipse is almost LP- $x$, while the degree of elliptical polarization is controlled by $\beta$. However, as the level of XPR is increased
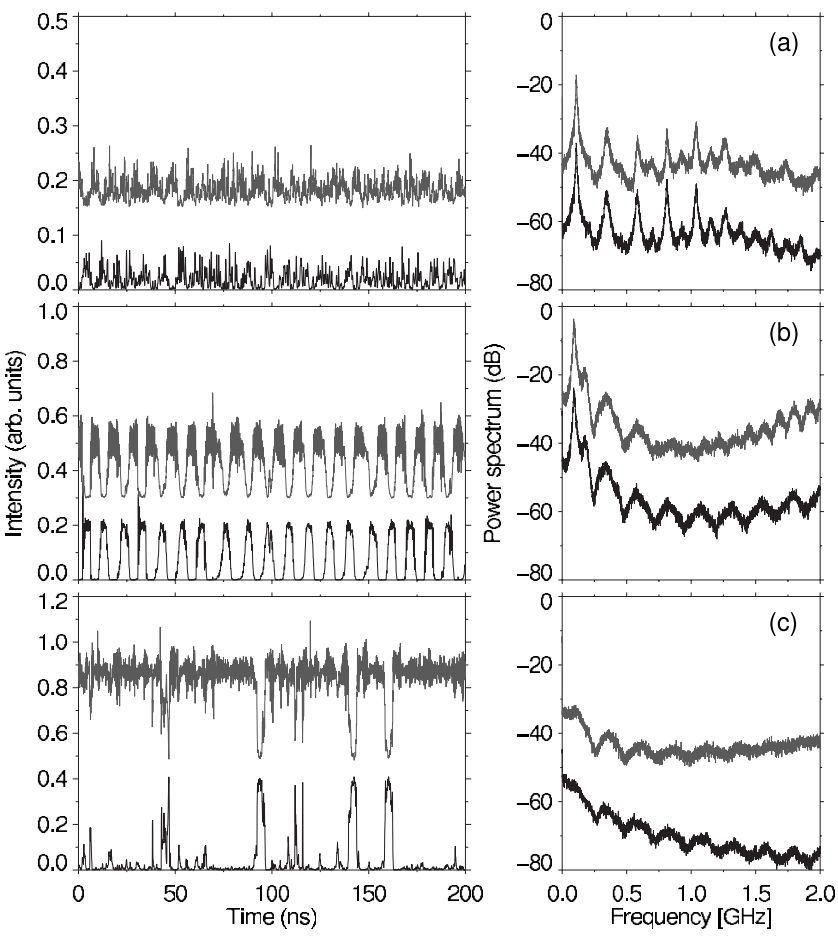

FIG. 6. (Left panels) Intensity of the LP-x (black) and LP-y (gray) components of the VCSEL for different currents: (a) $\mu$ $=1.05$, (b) $\mu=1.20$, and (c) $\mu=1.5$. The LP- $y$ component trace has been vertically shifted for the sake of clarity. (Right panels) The corresponding power spectra of the time traces. Parameters: Same as Fig. 5 except for $\beta=0.1$.

(upper left panel of Fig. 5), the emission in the elliptical state is destabilized and it displays an irregular behavior with irregularly distributed drops that correspond to a nascentalthough not yet developed-square waveform. These drops in the power of the LP- $x$ component are accompanied by intermittent bursts in the power of the LP- $y$, and the power spectra of both polarizations display weak and broad peaks. The fundamental frequency is slightly below $1 /(2 \tau)$ and the other peaks appear at odd multiples of the fundamental. These peaks also extend to higher frequencies (not shown in the figure) and they are more visible in the vicinity of the relaxation oscillation frequency. This corresponds to a slow modulation of the relaxation oscillations at almost twice the delay time in the reinjection loop.

Further increasing the level of XPR, the output in each linearly component becomes fully modulated by a squarewave of period $\sim 2 \tau$. The power spectrum is now dominated by the odd multiples of the fundamental frequency, although residual components at the even multiples can also be seen (see middle panels in Fig. 5). If the injection level is still increased, the quality of the square-wave switching deteriorates (lower panels in Fig. 5), and the power spectrum of the LP-y component exhibits high-frequency components close to $2 \gamma_{p}$ (not shown in the figure).

A very similar sequence of dynamical behaviors is observed when the level of XPR is kept constant and the current injection is varied (see Fig. 6). For low currents (upper panels) the output displays relatively large fluctuations with a 

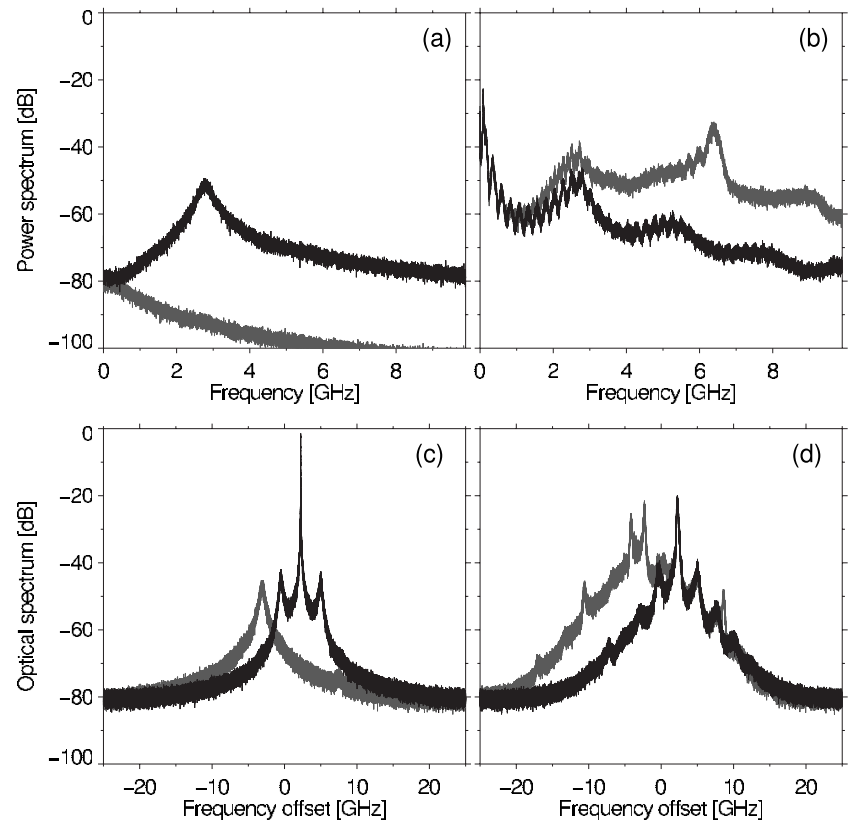

FIG. 7. (a), (b) Power and optical spectra for the solitary VCSEL and (c), (d) for a XPR level $\beta=0.1$. The current is set to $\mu=1.25$.

dominant frequency close to the relaxation oscillation frequency of the solitary VCSEL. As the pump current is increased, the square-wave modulation first becomes apparent (middle panels) but upon further increase of the current, the square-wave modulation of the output degrades and eventually disappears (lower panels).

The power and optical spectra corresponding to the solitary laser and the optimal XPR in the middle panel of Fig. 6 are represented in Fig. 7. In the absence of reinjection, the system operates on the LP- $x$ mode. Its power spectrum [see (a) displays only a broad peak that corresponds to the relaxation oscillations. These relaxation oscillations are also visible as sidebands in the optical spectrum [see (c)]. The LP-y component is strongly suppressed and its frequency splitting from the LP- $x$ is given by $\sim 2 \gamma_{p}$. In the presence of optimal XPR, the two power spectra exhibit well defined peaks at odd multiple of the fundamental $\sim 1 /(2 \tau)$. The power spectrum of the LP- $x$ component [see panel (b)] displays several harmonics of the relaxation oscillation since every period of the square switching regime, the LP- $x$ polarization starts from the off solution, thus resulting in a strongly nonlinear oscillation. This effect is also visible as sidebands on the optical spectrum of the LP- $x$ component [see (d)]. Similar features can be observed in the spectra of the LP-y component. However, we notice that the power spectrum of this component is strongly enhanced at a frequency close to 6.5 GHz. This frequency corresponds to the separation between the two main peaks in the optical spectrum, which occur at the frequencies of the peak of the dominant LP- $x$ component and the peak of the LP- $y$ amplified spontaneous emission in the solitary VCSEL.

The above results are in good agreement with the experimental observations, and they have been obtained for typical VCSEL parameter values. In addition, the values for $\beta$ also match those used in the experiment, and yield the observed phenomenology in the correct parameter ranges. We must however emphasize that the behavior obtained in the numerical simulations can be deeply affected by parameters choice. Indeed, this is also in qualitative agreement with the different behaviors observed from one device to the other.

The onset of the square-wave switching is mainly affected by cavity dichroism, birefringence and reinjection delay. Large values of $\gamma_{p}$ imply a reduced efficiency of XPR, since a larger detuning between the cavity resonances for LP- $x$ and the LP- $y$ polarizations leads to a smaller amplitude of the intracavity field and thus to a lower degree of cross-gain modulation. On the other hand, large dichroisms lead to a reduced amplification of the reinjected beam, thus also lowering the cross-gain saturation. On the other hand, for the square-wave regime to develop the delay has to be longer than the period of the relaxation oscillations. Instead, the degradation of the square wave regime depends mainly on the values of dichroism and spin-flip rate. As already commented, the degradation does not occur when the spinrelaxation rate is infinite. In addition, our numerical simulations indicate that the degradation is observed only for small dichroism such that the solitary VCSEL is weakly bistable or close to bistability. For large dichroism, the system is monostable and in this case, increasing the level of XPR always leads to an improvement of the quality of the squarewave switching in a similar way found in [8].

\section{THEORETICAL ANALYSIS}

The numerical evidences that we have provided so far are in good agreement with the experimental observations and they point to the important role played in our case by the mechanisms governing polarization in VCSELs. In the next subsections, we analyze in detail the spectrum of the monochromatic solutions, the onset of the square wave regime and its eventual deterioration.

\section{A. Monochromatic solutions}

The steady-state solutions of the SFM with XPR are defined by Eqs. (3)-(5), $E_{ \pm}(t)=A_{ \pm} e^{i \omega t}, N(t)=N_{\omega}$, and $n(t)=n_{\omega}$ and given by

$$
\begin{gathered}
i \omega A_{ \pm}=(1+i \alpha)\left(N_{\omega} \pm n_{\omega}-1\right) A_{ \pm}-\left(\gamma_{a}+i \gamma_{p}\right) A_{\mp} \\
\quad \pm i \frac{\beta}{2} e^{-i \omega \tau}\left(A_{+}+A_{-}\right), \\
0=\mu-N_{\omega}-\left(N_{\omega}+n_{\omega}\right)\left|A_{+}\right|^{2}-\left(N_{\omega}-n_{\omega}\right)\left|A_{-}\right|^{2}, \\
0=-\gamma_{s} n_{\omega}-\left(N_{\omega}+n_{\omega}\right)\left|A_{+}\right|^{2}+\left(N_{\omega}-n_{\omega}\right)\left|A_{-}\right|^{2} .
\end{gathered}
$$

This system admits in the first place a trivial solution $A_{ \pm}=0$, $N_{\omega}=\mu, n_{\omega}=0$ that corresponds to a nonlasing state. The linear stability analysis (LSA) of this solution reveals that it becomes unstable at $\mu=1-\left|\gamma_{a}\right|$, which corresponds to one of the lasing threshold of the LP- $x$ and LP- $y$ states. These thresholds are the same as in the solitary VCSEL, regardless of the level of XPR, as experimentally observed. 
In addition, the system also possesses a nontrivial solution $A_{-}=-A_{+}, \quad N_{\omega}=1-\gamma_{a}, n_{\omega}=0$, with $\left|A_{+}\right|^{2}=(1 / 2)\left[\mu /\left(1-\gamma_{a}\right)\right.$ $-1]$ which is identical to the $y$-polarized solution of the solitary VCSEL. In this state, which corresponds to lasing in the polarization that is suppressed by the linear polarizer after the polarization rotator, the system does not experience any XPR. Consequently, this solution is independent of $\beta$ and, more surprisingly, its primary bifurcation sequence is exactly the same as that in the solitary VCSEL, with the same bifurcation points. However, as we shall discuss later, the type of the existing Hopf bifurcations can be modified by XPR, passing, in some parameter ranges, from subcritical to supercritical.

Finally, the system possesses a family of elliptically polarized monochromatic states with $n_{\omega} \neq 0$ and $A_{+} \neq 0 \neq A_{-}$, which are given by

$$
\begin{gathered}
1=K_{+} K_{-}, \\
N_{\omega}=\mu-\left[N_{\omega}+n_{\omega}+\left(N_{\omega}-n_{\omega}\right)\left|K_{+}\right|^{2}\right]\left|A_{+}\right|^{2}, \\
n_{\omega}=-\frac{1}{\gamma_{s}}\left[N_{\omega}+n_{\omega}-\left(N_{\omega}-n_{\omega}\right)\left|K_{+}\right|^{2}\right]\left|A_{+}\right|^{2}, \\
K_{ \pm}=\frac{\gamma_{a}+i \gamma_{p} \pm i \beta / 2 e^{-i \omega \tau}}{i \omega-(1+i \alpha)\left(N_{\omega} \mp n_{\omega}-1\right) \pm i \beta / 2 e^{-i \omega \tau}}, \\
A_{-}=-K_{+} A_{+} .
\end{gathered}
$$

Surprisingly, although XPR corresponds to what is often denoted as "incoherent feedback," Eqs. (9)-(13) may have multiple solutions that look very much like the external cavity modes found in conventional optical feedback. The reason is that for any finite value of the spin-flip rate $\gamma_{s}$ there is a coherent coupling between $E_{x, y}$ of strength given by $n|\beta| \sqrt{1+\alpha^{2}}$, which vanishes only when $n \equiv 0$. This coherent coupling allows for multiple solutions of different degree of ellipticity, different total power and different frequency to appear, as shown in Fig. 8(a). These modes have different frequencies and are located around the solitary LP- $x$ frequency $\gamma_{p}$ on a twisted curve $\epsilon_{x}$. The isolated point around $-\gamma_{p}$ corresponds to the LP- $y$ solution. The projection of $\epsilon_{x}$ onto the $(\omega, N)$ plane shows a remarkable similarity with the so-called external cavity modes of the Lang-Kobayashi model [18]. The spin-flip rate $\gamma_{s}$ and the reinjection $\beta$ fully control the aperture of the "ellipse." Either in the limit $\gamma_{s}$ $\rightarrow+\infty$ or $\beta \rightarrow 0$ the ellipse collapses onto the LP- $x$ solution. As a supplementary analogy with the Lang-Kobayashi model, new solutions are born in pairs via saddle-node bifurcation as shown in Fig. 8(b) but they are not branched on the nonlasing solution. It is worth noticing the small discrimination in the total inversion $N$ among these solutions, meaning that they are almost degenerate in power.

\section{B. Onset of the square wave regime}

The square wave regime develops from the elliptically polarized solution with lowest threshold through a complicate sequence of bifurcations with the XPR level. In spite of
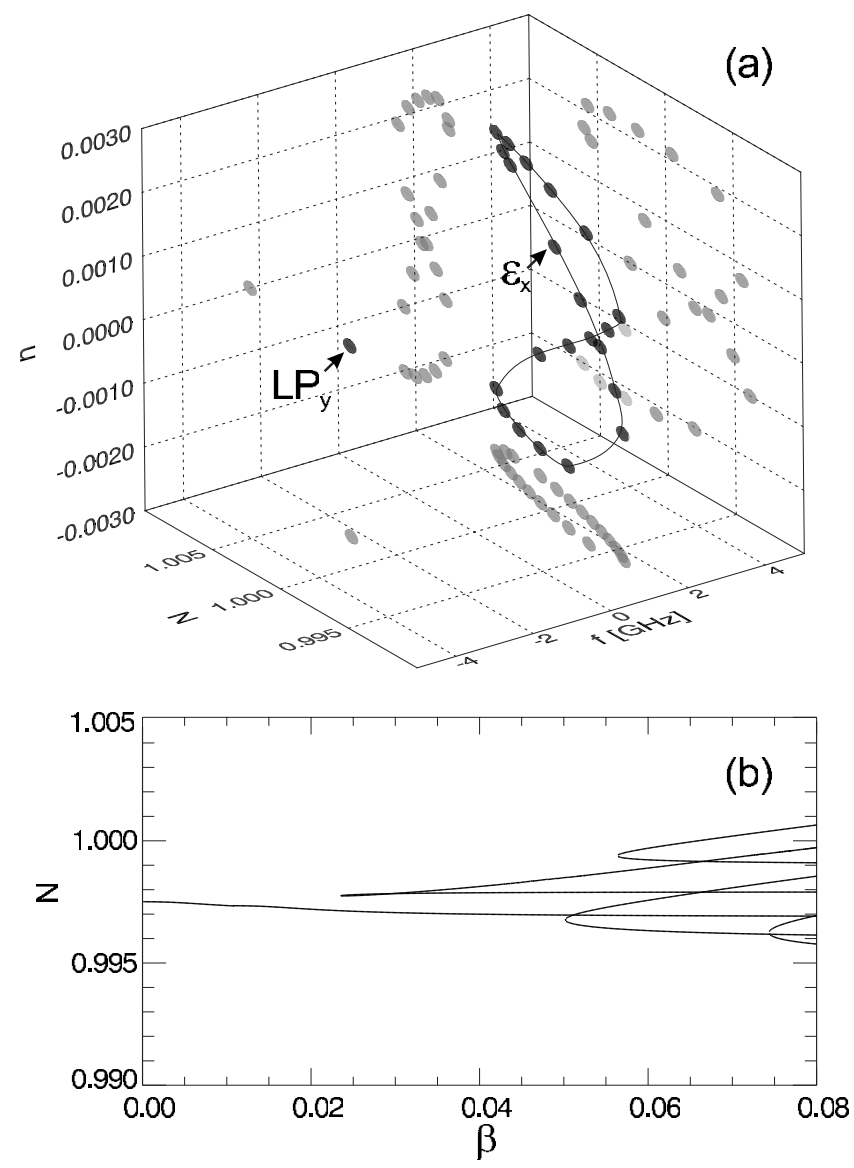

FIG. 8. (a) Steady states solution of Eqs. (9)-(13) in the $(\omega, N, n)$ projection for $\beta=0.25$. (b) Bifurcation diagram as function of the reinjection rate $\beta$. In both cases, the bias current is $\mu=1.25$.

this complicated bifurcation sequence, the onset of the square-wave switching can still be understood in terms of the analysis of the plateaux performed in [8] which is valid when $\tau$ is much longer than any other internal time scale of the system, so transients at the beginning of each plateau can be disregarded.

In this analysis, the square wave regime corresponds to a period-two solution on the slow time scale $\tau$, thus describing a quasistatic orbit between two distinct stages. The former stage corresponds to LP- $x$ emission and the latter to amplification of the reinjected light in LP- $y$. Both stages last for a time $\sim \tau$ that corresponds to the flight time in the reinjection arm. Two necessary conditions have to be satisfied for this regime to exist. First, the arrival of the XPR light has to suppress the gain for lasing in LP- $x$. The second condition is that the solitary laser be unable to self-sustain emission in LP- $y$.

The first condition requires that the population inversion during the second stage has to be smaller than the laser threshold. When this condition is not fulfilled, LP- $x$ emission is only partially suppressed by XPR. Accordingly, at the end of the second plateau, the LP- $y$ component may receive this weakened LP- $x$ emission which may initiate a third plateau, thus destroying the period two cycle. On the other hand, if the second condition is not met, the system may remain trapped in the LP- $y$ state. 
According to the above discussion, we proceed finding the solution of Eqs. (3)-(5) for each plateau. The state of the system during the first plateau is

$$
\left(E_{x}, E_{y}, N, n\right)=\left(\rho_{1} e^{i \omega_{1} t}, 0, N_{1}, 0\right),
$$

with $\rho_{1}^{2}=\left(\mu-N_{1}\right) / N_{1}$ and $\omega_{1}=-\gamma_{p}+\alpha \gamma_{a}$ while the inversion corresponds to the threshold of the LP- $x$ solution $N_{1}=1+\gamma_{a}$. On the other hand, the state during the second plateau is given by

$$
\left(E_{x}, E_{y}, N, n\right)=\left(0, \rho_{2} e^{i\left(\omega_{1} t+\phi\right)}, N_{2}, 0\right),
$$

with $N_{2}=\mu /\left(1+\rho_{2}^{2}\right)$. This condition provides a relationship for finding $\rho_{2}$ which reads

$$
i \omega_{1}=(1+i \alpha)\left(N_{2}-1\right)+z+\beta \frac{\rho_{1}}{\rho_{2}} e^{-i\left(\omega_{1} \tau+\phi\right)} .
$$

The locus of the second plateau possesses a strong similarity with the defining relation of a laser with injected signal, that can exhibit bistability. However, the condition $N_{2}$ $\leqslant N_{1}$ does not allow for this possibility. We can estimate the critical value of the reinjection from $N_{2}\left(\beta^{\star}\right)=N_{1}$, which determines $\left|\beta^{\star}\right|$ as

$$
\left|\beta^{\star}\right|=2 \sqrt{\gamma_{a}^{2}+\gamma_{p}^{2}} .
$$

When $\beta<\beta^{\star}$, XPR is not sufficient to suppress LP- $x$ emission during the second stage and an incomplete, noisetriggered square wave switching is found. With our choice of parameters, $\beta^{\star} \sim 0.105$, which closely matches the optimal regime.

\section{Degradation of the square waves}

One of the characteristic features of our system is that the square waveform deteriorates when either the level of XPR or bias current are increased too much. In this section, we analyze the underlying mechanisms that govern this phenomenon.

As stated in the previous subsection, a necessary condition for the square-wave switching regime to exist is that the system has to be unable to sustain emission in the LP-y mode. Therefore, mechanisms changing the stability of the LP- $y$ mode will play an important role. In particular, the onset of bistability, as it is often encountered in VCSELs as current is increased, should imply the disappearance of the squares.

Accordingly, we start by analyzing the linear stability (LSA) of the LP- $y$ solution. The dynamical evolution of the perturbations around the LP-y solution is governed by the equations

$$
\dot{S}=2(1+i \alpha) Q \Delta+i \frac{\beta}{2} R(t-\tau) e^{-i\left(\gamma_{p}-\alpha \gamma_{a}\right) \tau},
$$

$$
T \dot{\Delta}=-\mu \Delta-Q\left(S+S^{*}\right)
$$

$$
\dot{R}=2(1+i \alpha) Q \delta-2\left(\gamma_{a}+i \gamma_{p}\right) R
$$

$$
T \dot{\delta}=-\left(\gamma_{s}+2 Q^{2}\right) \delta-Q\left(1-\gamma_{a}\right)\left(R+R^{*}\right),
$$

where $S, R, \Delta$, and $\delta$ stand for the deviations from the LP-y steady state for the total field, the field difference, the total carrier density, and the difference of carrier densities between the two spin channels, respectively (See Ref. [15] for more details.) In addition, we have defined the total power as $Q^{2}=(1 / 2)\left[\mu /\left(1-\gamma_{a}\right)-1\right]$. In the above system we can identify two blocks. The first one, $\left\{S, S^{*}, \Delta\right\}$, describes the classical relaxation oscillation between the total field and the population inversion. The second one, $\left\{R, R^{*}, \delta\right\}$, governs the polarization dynamics and it is the responsible of changes in polarization stability. For a solitary VCSEL where the dominant mode is on the blue side of the frequency spectrum, i.e., $\left(\gamma_{a}, \gamma_{p}\right)<(0,0)$, the main mechanism allowing the unstable LP- $y$ mode to regain stability [17] is an Andronov-Hopf bifurcation with an oscillation frequency $\omega_{h} \sim 2 \gamma_{p}$. In the presence of reinjection, one can clearly see that this second block is not modified, thus bifurcations connected to the LP-y mode that exist in the solitary VCSEL remain unchanged in the presence of XPR. In a similar way, the first block, that represents the usual damped relaxation oscillations in class B lasers, cannot be responsible of any bifurcation, since reinjection appears only as an external forcing term in Eq. (18). Accordingly, the eigenvalues and the possible local bifurcations are not modified by the presence of XPR which can only affect the form of the unfolding.

In order to analyze the effects of reinjection onto the bifurcation characteristics, we employed DDEBifTool [19] which allows to track stable and unstable periodic solutions. The bifurcation diagrams for different values of XPR are represented in Fig. 9. With our choice of parameters, a Andronov-Hopf bifurcation occurs at $\mu_{h} \sim 1.285$. Above this current, the LP- $y$ solution is stable regardless of the value of $\beta$.

For very low XPR, see curve (b) in Fig. 9, a subcritical bifurcation occurs when $\mu$ is increased above $\mu_{h}$. The nascent limit cycle is unstable and separates the LP-y solution from the elliptical states. When the reinjection reaches $\beta$ $=0.03$, see curve (c), the direction of the limit cycle is reversed, thus the bifurcation becomes supercritical and the system exhibits a region of periodic intensity pulsations for currents below $\mu_{h}$ but above the folding point of the supercritical branch, $\mu_{f}$. The nascent limit cycle is stable in some current range above the folding point but below $\mu_{h}$, and it coexists with the other possible dynamical states. Further 


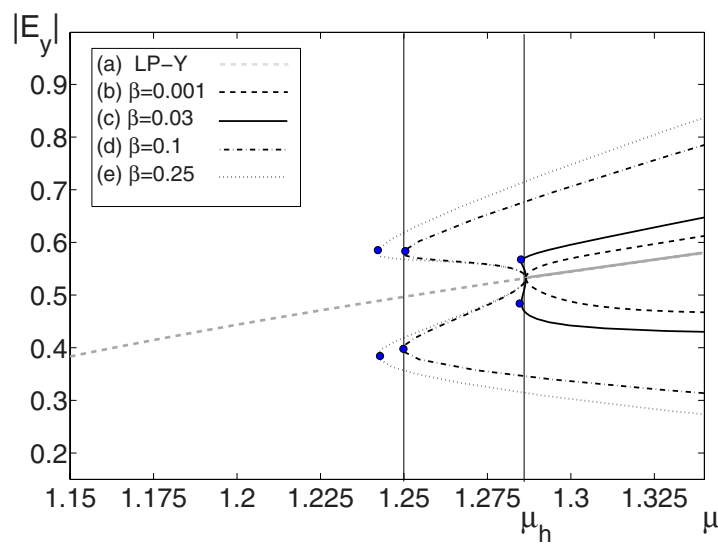

FIG. 9. (Color online) Bifurcation diagram of the LP- $y$ solution as a function of $\mu$ for different reinjection rate $\beta$. The LP- $y$ mode is stable after the bifurcation point. The supercritical quasiperiodic solutions $[(\mathrm{c}),(\mathrm{d})$, and (e)] are stable in some current range below $\mu_{h}$. The circles denote the folding point $\mu_{f}$ for the supercritical branches.

increasing the reinjection pushes $\mu_{f}$ deeper in the low current region, thus enlarging the region of coexistence; see curves (d), (e).

The above diagram allows us to understand the observed degradation of the square-wave switching for large values of current or reinjection. For a fixed current below $\mu_{h}$, the square waveform develops for $\beta>\beta^{\star}$ and it becomes progressively more regular upon increasing $\beta$. At the same time, increasing $\beta$ decreases the value of $\mu_{f}$ until eventually it coincides with the bias current applied to the VCSEL. From this point on, the dynamics of the system will be changed because the system will spend some time in the vicinity of the new, stable limit cycle causing a deterioration of the timing of the square waveform. Further increases of $\beta$ render the limit cycle (or the attractor that develops from it) more stable, thus eventually leading to the complete degradation of the square-wave regime. This is exemplified by the vertical line in Fig. 9 at $\mu=1.25$ : The folding point touches it for $\beta$ $\gtrsim 0.1$; that corresponds to the optimum value for the squares in Fig. 5. At this current value, for $\beta=0.25$, the limit cycle is well developed and the square-wave switching has disappeared (see Fig. 5). It is worth noticing that, since the folding point is almost insensitive to variations of the reinjection phase, this mechanism is thus robust, in agreement with our experimental findings. In addition, the frequency of oscillation of this limit cycle is close to $2 \gamma_{p}$, in agreement with the peaks obtained in the optical spectra. In the same way, for a fixed level of XPR above $\beta^{\star}$, the square-wave regime deteriorates when the current is increased beyond the value that corresponds to the folding point of the two periodic branches. In particular, the folding of the periodic branches explains the numerical observation of degradation of the square-wave regime at current values slightly below the boundary of bistability of the solitary laser.

\section{CONCLUSIONS}

In conclusion, we have studied both experimentally and theoretically the effects of crossed-polarization reinjection in a VCSEL. We have shown that for XPR levels above a certain critical value, the output of the system exhibits squarewave modulation at a frequency corresponding to the inverse of twice the reinjection delay, as previously observed in edge emitters. However, we have observed that in VCSELs the square-wave switching is optimal in specific ranges of current and levels of XPR. If the level of XPR is too strong, the square-wave switching degrades, and the same happens if the current is either too low or too high.

We have explained these results within the framework of the spin-flip model, and we have shown that, in spite of the fact that XPR is usually denoted as incoherent feedback, it gives rise to a whole family of elliptically polarized states of different power, ellipticity and frequency. These states arise due to the coherent coupling of the field components mediated by the difference in carrier density between the two spin channels.

Necessary conditions for the onset of the square waves have been obtained considering the squares as a switching between two steady plateaux, which provides the minimum value of XPR level for the square waves to exist.

The degradation of the square-wave switching upon increasing of either the reinjection level or current value is related to the finite value of the spin relaxation rate. Degradation occurs through the stabilization of a periodic solution arising from the Hopf bifurcation of the LP-y mode. This bifurcation becomes supercritical for high enough reinjection values, and induces the deterioration of the square-wave regime when the system is operated near its folding point. In the parameter regimes where only one component is strongly favored, degradation of the square-wave switching was not observed.

\section{ACKNOWLEDGMENTS}

This work has been funded through the project TEC2006-13887-C05-03/TCM from the Spanish Ministerio de Educación y Ciencia. J.J. acknowledges support from the program Juan de la Cierva, Ministerio de Educación y Ciencia. M.G. acknowledges partial financial support from Universitat de les Illes Balears.
[1] E. P. Ippen, Appl. Phys. B: Lasers Opt. 58, 159 (1994).

[2] P. P. Vasil'ev, I. H. White, and J. Gowar, Rep. Prog. Phys. 63, 1997 (2000).

[3] H. Yasaka, Y. Yoshikuni, and K. Kawaguchi, IEEE J. Quantum
Electron. 27, 193 (1991).

[4] S. Jiang, Z. Pan, M. Dagenais, R. A. Morgan, and K. Kojima, Appl. Phys. Lett. 64, 3545 (1993).

[5] H. Li, A. Hohl, A. Gavrielides, H. Hou, and K. D. Choquette, 
Appl. Phys. Lett. 72, 2355 (1998).

[6] T. Heil, A. Uchida, P. Davis, and T. Aida, Phys. Rev. A 68, 033811 (2003).

[7] J. Houlihan, G. Huyet, and J. G. McInerney, Opt. Commun. 199, 175 (2001).

[8] A. Gavrielides, T. Erneux, D. W. Sukow, G. Burner, T. McLachlan, J. Miller, and J. Amonette, Opt. Lett. 31, 2006 (2006).

[9] M. San Miguel, in Semiconductor Quantum Optoelectronics, edited by A. Miller, M. Ebrahimzadeh, and D. Finlayson (Institute of Physics, Bristol, 1999), p. 339.

[10] M. Sondermann, T. Ackemann, S. Balle, J. Mulet, and K. Panajotov, Opt. Commun. 235, 421 (2004).

[11] F. Marino, L. Furfaro, and S. Balle, Appl. Phys. Lett. 86, 151116 (2005).
[12] J. Javaloyes, J. Mulet, and S. Balle, Phys. Rev. Lett. 97, 163902 (2006).

[13] M. San Miguel, Q. Feng, and J. V. Moloney, Phys. Rev. A 52, 1728 (1995).

[14] C. H. Henry, IEEE J. Quantum Electron. 18, 259 (1982).

[15] J. Martín-Regalado, F. Prati, M. San Miguel, and N. Abraham, IEEE J. Quantum Electron. 33, 765 (1997).

[16] J. Mulet, C. R. Mirasso, and M. San Miguel, Phys. Rev. A 64, 023817 (2001)

[17] T. Erneux, J. Danckaert, K. Panajotov, and I. Veretennicoff, Phys. Rev. A 59, 4660 (1999).

[18] R. Lang and K. Kobayashi, IEEE J. Quantum Electron. 16, 347 (1980).

[19] K. Engelborghs, T. Luzyanina, and D. Roose, ACM Trans. Math. Softw. 28, 1 (2002). 